\title{
Aportes colombianos a la tiroidología
}

\section{Alfredo Jácome Roca*}

${ }^{*} M D$, FACP. Internista-Endocrinólogo. Miembro de Número, Academia Nacional de Medicina de Colombia, Bogotá. Miembro Honorario, Asociación Colombiana de Endocrinología, Diabetes y Metabolismo.

\section{Resumen}

$\mathrm{L}$ as patologías tiroideas estudiadas inicialmente desde la época de Mutis fueron el bocio y el cretinismo endémicos. Una segunda etapa le correspondió a los endocrinólogos de la segunda mitad del siglo XX, que ampliaron los conocimientos etiológicos, patológicos, diagnósticos y terapéuticos, destacándose la investigación de agentes bociogénicos en el Valle del Cauca y la implementación de los estudios y terapéuticas con yodo radioactivo. El mejor conocimiento de estos pacientes hizo reducir las tiroidectomías a unas proporciones más racionales, mientras que programas de salud pública como la yodación de la sal y la detección rutinaria de hipotiroidismo congénito por medio de la TSH neonatal lograron un gran avance en la prevención y pronóstico de estas patologías. Actualmente se adelantan investigaciones genéticas, de técnicas quirúrgicas y de autoinmunidad, al tiempo que el país participa en estudios multicéntricos regionales sobre patologías tiroideas.

Palabras clave: hipertiroidismo, hipotiroidismo, cáncer de tiroides, bocio endémico, yodación de la sal, autoinmunidad tiroidea, tiroides Colombia.
Abstract
Thyroid pathologies such as endemic goiter and cretinism were studied by Spanish scientist Jose Celestino Mutis during the Colonial era in Colombia. Twentieth century endocrinologists conducted research that contributed to expand our knowledge on the etiol- ogy, histopathology, diagnosis and therapy of thyroid diseases. Two advances to highlight is the discovery of goitrogens in the Valley of the Cauca River and the use of radioactive iodine for diagnostic and therapeutic purposes. A better knowledge of patients with thyroid diseases resulted in a decrease in the number of thyroidectomies to reasonable proportions, whereas public health programs such as salt-iodination and routine screening of congenital hypothyroid- ism via neonatal TSH, achieved significant advances in the preven- tion and prognosis of these pathologies. Current research is aimed at genetics, better surgical techniques and thyroid autoimmunity, while Colombian scientists are increasingly taking part in regional multi-center studies.

Key words: Hyperthyroidism, hypothyroidism, thyroid cancer, radioactive iodine, endemic goiter, salt iodination, thyroid autoimmunity, thyroid Colombia.

\section{Introducción}

El bocio y el cretinismo endémicos fueron la primera y más evidente preocupación sanitaria de la región andina en relación con la glándula tiroides. "Una enfermedad horrorosa", dijo Jean-Baptiste Boussingault, quien sugirió el tratamiento apropiado $^{(1)}$. Habría de pasar más de un siglo antes de que se implementara la yodación de la sal, como programa lógico de salud pública. La detección neonatal del hipotiroidismo congénito en Colombia se realiza con fines preventivos y la enfermedad tiroidea autoinmune tiene consecuencias más allá de la misma glándula, pues es además un importante marcador de otras patologías autoinmunes. La disfunción tiroidea tiende a comprometer la viabilidad de los embarazos o a producir graves complicaciones cardiovasculares. El estudio del cáncer tiroideo es importante desde el punto de vista epidemiológico, del diagnóstico precoz y de la detección del carcinoma medular en familias, ya que además puede asociarse con tumores en diversos tejidos endocrinos; también en nuestro país se están haciendo importantes aportes sobre nuevas técnicas quirúrgicas. Estas patologías que hemos nombrado constituyen un problema mundial en general y colombiano en particular; por esto resulta necesario evitar que los programas de vigilancia existentes sean echados al olvido, por las repercusiones que esto tendría sobre la salud de los connacionales y por la importancia de la investigación en la información epidemiológica. En este orden de ideas, realizamos una valoración de la literatura colombiana sobre tiroidología, recordando la que hicimos en $1978^{(2)}$, con el objeto de determinar el nivel de producción en esta materia.

\section{Material y métodos}

Con el objeto de localizar los estudios sobre tiroides realizados en nuestro país, se hizo una búsqueda de la literatura indizada en las bases de datos de PubMed (1961-2013) para publicaciones en inglés, y de LILACS (1959-2012), SciELO Colombia (2004-2013) e IMBIOMED (2005-2012) para los trabajos en español. Es de anotar que en esta última base de datos, no todas sus revistas aparecen también en SciELO. Se incluyeron todos los tipos de estudios caracterizándolos por años, 
tipo de publicación y autores con mayor calidad y número de aportes, usando como palabras clave las de hipertiroidismo, hipotiroidismo, cáncer de tiroides, bocio endémico, yodación de la sal y autoinmunidad tiroidea. Estos términos interactuaron (AND) con la palabra Colombia. Se consultaron también varios libros y documentos, así como material impreso de algunas revistas no incluidas en dichas bases de datos. Al final se incluyeron para análisis los artículos de investigación original y algunos informes clínicos, considerados por el autor como los más importantes.

\section{Resultados}

En MedLine/PubMed se encontraron 58 artículos (entre 156.711 artículos totales sobre tiroidología, a partir de 1961), de los cuales 31 fueron entre 2007 y 2013. Investigaciones originales fueron 35, 27 de ellas publicadas en inglés. Un total de 10 fueron informes de casos y 7, revisiones. De los artículos listados en PubMed, 48 se escribieron en inglés (de los cuales 34 fueron observaciones en seres humanos, pero sólo 7 de ellos fueron publicados en las revistas de más alto impacto) y 8 en español (7 en humanos, en revistas de un cuartil más bajo). De 39 artículos en los últimos 11 años, 12 fueron sobre autoinmunidad y 13 sobre cáncer). En los últimos 6 años, de 28 trabajos sobre autoinmunidad o cáncer, las 3/5 partes fueron sobre este último tema. Buscando "bocio endémico" y "Colombia" como palabras claves relacionadas, aparecieron 15 artículos internacionales, pero sólo dos cuando se utilizó la palabra "cretinismo", indicando la disminución del problema, merced a los programas de la yodación de la sal de cocina y la detección de casos de hipotiroidismo neonatal. La productividad en los últimos 10 años se duplicó (para llegar a 9 artículos en el último año). Hubo superposición en los títulos de algunos trabajos que aparecieron también en otras bases de datos.

A partir del año 2000, se encontró una sola referencia en revistas médicas de alto impacto ${ }^{(4)}$. Ya en la totalidad del universo estudiado, 5 de los 7 estudios publicados en las revistas consideradas de alto impacto por Medline/PubMed correspondieron al grupo de Cali (siglos XX y XXI). Una vez emigraron los principales miembros del grupo investigadores de estos temas en la Universidad del Valle, se nota una mayor dispersión de los centros universitarios donde se realizaron trabajos que aparecieron en revistas acogidas en la lista de PubMed, o que aparecen en las restantes bases de datos enunciadas.

La gran mayoría de artículos indexados se originaron en Bogotá, Medellín y Cali, siendo la revista más utilizada el Journal of Clinical Endocrinology \& Metabolism, aunque sólo en cuatro ocasiones. Los demás artículos se encuentran publicados en diversas revistas extranjeras de inmunología, reumatología, de cáncer y de tiroides, siendo Biomédica la única publicación colombiana incluida. En PubMed, el autor que más contribuyó fue Juan Manuel Anaya (Universidad del Rosario), con 9 artículos, seguido de Eduardo Gaitán Marulanda (Universidad del Valle, Cali) con 8, Álvaro Sanabria con 7 (Universidad de la Sabana) y Adriana Rojas-Villarraga (Universidad del Rosario) con 6, Pelayo Correa (Universidad del Valle) con 5, y Ángela Londoño (Universidad del Quindío), 3. En la lista de Lilacs (desde que se empezó a elaborar esta base de datos), aparecen 40 citaciones, pero 5 de ellas son de tipo veterinario. Es de anotar que en la Revista de la Sociedad Colombiana de Endocrinología - que fue publicada durante varios lustros antes de desaparecer y no quedó incluida en la base de datos de LILACS - aparecieron varios artículos de grupos interesados en la tiroidología, como los del Instituto Nacional de Cancerología, Hospital San José, Universidad de Antioquia y, por supuesto, de la Universidad del Valle. La base de datos de SciELO muestra 10 artículos, en las revistas Biomédica, Colombia Médica, Salud Pública, revistas de España, Chile y OPS, incluidas en PubMed. De las no incluidas se prefirió a la Revista de la Sociedad Colombiana de Cirugía con 3 artículos, y uno de cada uno en Acta Médica Colombiana, Salud Uninorte y la Revista de la Facultad de Medicina de la Universidad Nacional. La base de datos de Imbiomed, que tiene 219 registros de la palabra tiroides, contiene alrededor de un $10 \%$ de artículos generados en nuestro país.

Es de anotar que todos los estudios colombianos sobre tiroides se buscaron en las bases de datos, pero muchos no fueron incluidos entre las referencias, para no hacer demasiado larga la lista.

\section{La tiroidología en Colombia}

A finales del siglo XIX y comienzos del XX, se fueron conociendo las enfermedades tiroideas. Entonces el interés de los médicos se expandió: se hicieron cirugías, se prescribía un preparado de tiroides desecado y se empezó a usar el metabolismo basal como prueba de función tiroidea ${ }^{(1-5)}$.

En la segunda mitad del siglo XX, se mejoraron las pruebas diagnósticas, la terapia de suplencia se dirigió hacia preparados confiables de levotiroxina y — para la valoración del nódulo tiroideo- se pasó de la biopsia tru-cut al aspirado citológico con aguja fina que, en manos de un citólogo experimentado, resulta de gran ayuda para el tratamiento del cáncer de tiroides, implementándose además la terapéutica con yodo radioactivo. Pero la mejor prueba de función tiroidea ha resultado indudablemente la TSH ultrasensible, con base en la cual han mejorado los criterios de tratamiento.

\section{Bocio y cretinismo endémicos}

Boussingault era un ingeniero de minas y científico pionero de la química agrícola en el siglo XIX, quien por serendipia descubrió la relación del bocio con la deficiencia de yodo en las cordilleras andinas. El francés había sido traído por Bolívar y Santander a través de Francisco Antonio Zea para estudios 
sobre la minería, lo que le permitió la observación de numerosos campesinos cotudos y cretinos en las áreas montañosas que visitó. Según Rueda-Williamson (quien estuvo a cargo de la ejecución inicial del plan para erradicar el bocio endémico) "el eminente sabio francés publicó sus observaciones en las Memorias sobre las salinas yodíferas de los Andes y las causas del coto en las cordilleras de la Nueva Granada ${ }^{(1)}$. Con increíble visión de medicina preventiva para su tiempo, afirmaba entonces que "el bocio desaparecería de la Nueva Granada (hoy Colombia) si las autoridades tomaran medidas para establecer en cada cabecera de cantón donde el coto es endémico, depósitos de sales yodíferas y en las que los habitantes pudieran surtirse de la sal necesaria para su consumo". En Antioquia no había coto, pues la sal la tomaban de fuentes saladas de rocas cristalinas ricas en yodo como en Heliconia.

La yodación de la sal de consumo cotidiano redujo de manera significativa la problemática sanitaria del cretinismo endémico, estudiado por endocrinólogos colombianos en 1959 que ganaron el Premio de la Fundación Alejandro Ángel Escobar en $1959^{(6)}$. Este mismo premio lo obtendría luego Eduardo Gaitán, quien en 1976 presentó su trabajo “Distribución, naturaleza y fuentes de origen de los agentes bociogénicos en el occidente

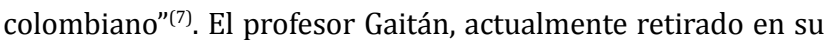
casa del Valle del Cauca, es el autor de una de las bibliografías mundiales más importantes sobre este tema ${ }^{(7-9)}$. En un reciente artículo "se encontró que en Colombia el diagnóstico de deficiencia de yodo (DDY) es subvalorado, desconociéndose la problemática actual y el estado del yodo poblacional, por lo que es necesario realizar estudios, ya que es evidente el abandono político de este problema prevenible, predecible y tratable. La mejor forma de evaluar los DDY es por medio de la yoduria y la mejor estrategia para reducir la prevalencia de estos desórdenes en la población es a través de la yodación universal de la sal"(10). Existen regiones en el país en donde la vigilancia de la ingesta de yodo no es adecuada, corriéndose el riesgo de reaparición de casos de bocio/cretinismo endémicos.

Hace más de tres décadas los endocrinólogos editamos un libro sobre la Tiroidología en Colombia ${ }^{(2)}$ que recogía investigaciones y revisiones nuestras de aquellas épocas. Además de los elegantes estudios de Eduardo Gaitán, están las publicaciones del grupo del Instituto de Cancerología en relación con los estudios con yodo radioactivo de la función tiroidea, de la glándula cancerosa o nodular benigna ${ }^{(11)}$. Se incluyó también información continuada del estudio llevado a cabo por un grupo de endocrinólogos colombianos sobre factores etiológicos del cretinismo endémico, que fue premiado en 1959 por la Fundación Alejandro Ángel Escobar ${ }^{(6)}$. Llevado a cabo principalmente en Mariquita y en la Sierra Nevada, demostró el efecto benéfico de la yodación en la mayoría de los casos pero no en todos, por lo que se concluyó que debían existir otros bociógenos importantes en el agua o en los alimentos. De aquellas épocas son también la obra del profesor Patiño, sobre bocio y cáncer de tiroides ${ }^{(12)}$ y algunos libros que narran episodios que dieron lugar al nacimiento de la endocrinología en Colombia, y a su madurez en la segunda mitad del siglo $\mathrm{XX}^{(2,4,5)}$.

Hay que hacer un reconocimiento especial al papel que jugaron los isótopos de yodo - en particular el I-131- en el estudio y tratamiento de las enfermedades tiroideas. Grupos de Bogotá, Cali y Medellín lograron estudiar numerosos pacientes, y adquirir experiencia en cifras normales, imaginología y terapéutica en cáncer y en hipertiroidismo ${ }^{(13,14)}$. Pruebas diagnósticas clásicas, como el metabolismo basal, la yodoproteinemia y la captación de yodo radioactivo en 24 horas fueron remplazadas por la TSH ultrasensible, la T4 total y libre, la T3 total y la captación de T3, con métodos de radioinmunoanálisis y ELISA; el isótopo I-131 fue cambiado por el más ventajoso Tc99 para procedimientos gammagráficos tiroideos, emergiendo la ecografía de alta resolución y otros procedimientos imaginológicos en el moderno estudio de la glándula. Para algunos casos de hipotiroidismo, la prueba de TSH pos-TRH y la medición de tiroglobulina sérica como marcador de cáncer de tiroides. La biopsia por aspiración con aguja fina es definitiva en la potencial decisión quirúrgica en el estudio de los nódulos tiroideos, mientras que la biopsia por congelación continúa siendo confiable para muchos cirujanos de cabeza y cuello, para tomar decisiones intraoperatorias sobre la extensión de la tiroidectomía.

\section{Hipotiroidismo congénito}

Los recién nacidos en áreas bociosas con adecuada suplementación de yodo tienen un funcionamiento de glándula tiroides similar al de los niños nacidos en áreas no bociosas con la misma suplementación de yodo y no tienen, por lo tanto, riesgo más alto de desarrollar hipotiroidismo congénito ${ }^{(15)}$. En 1998 Colombia fue declarada país libre de desórdenes por deficiencia de yodo. Sumado a esta estrategia, la resolución 412 del 2000 adoptó como norma el tamizaje neonatal para hipotiroidismo congénito en la atención al recién nacido ${ }^{(16)}$. A partir de 1979 , un grupo de trabajo de la Facultad de Medicina de la Universidad Nacional comenzó el desarrollo de la primera experiencia de detección neonatal a través del programa de hipotiroidismo congénito, el cual se inició en el Instituto Materno Infantil de Bogotá y permitió en una primera fase la constitución de un grupo colaborativo con otras entidades del país, y en una segunda fase, comprendida entre 1983 y 1985, permitió tamizar 10.202 neonatos, confirmándose en cuatro de ellos el diagnóstico de hipotiroidismo congénito, lo que correspondió a una incidencia de $1: 2.500^{(17,18)}$. De acuerdo con estudios de la Universidad Javeriana de Bogotá, se tamizaron desde 1983 hasta 1998 diecisiete mil (17.000) recién nacidos, en los cuales se detectaron doce casos de hipotiroidismo congénito, cuatro de los cuales fueron transitorios, lo que equivale a una frecuencia de 1:2.100 ${ }^{(19)}$.

Diferentes programas colombianos de tamizaje han indicado una incidencia entre 1:536 y 1:3600 recién nacidos en las series individuales de casos por laboratorio o por institución de 
salud $^{(20,21)}$. El protocolo de la Red Nacional de Laboratorios ${ }^{(22)}$ para el tamizaje neonatal de hipotiroidismo congénito y la vigilancia por laboratorio ha permitido estandarizar la técnica y las metodologías. En la actualidad, 152 laboratorios públicos y privados participan de forma rutinaria en el "programa de evaluación externa del desempeño para la prueba de TSH en muestras de sangre seca de cordón umbilical". Para el 2005 se informaron resultados de laboratorio de 589.490 muestras, lo que indicaría una cobertura cercana al $70 \%$ de todos los nacimientos para ese año. Sobre esa muestra se identificaron 157 casos que representan una incidencia de uno por cada 3.755 niños. Para el 2007, solamente el Distrito Capital logró coberturas superiores al 95\%, incluida la población pobre no asegurada, la cual constituye el principal problema de cobertura, aun en los departamentos con mejores coberturas por parte de las aseguradoras tanto del régimen contributivo como del subsidiado ${ }^{(23)}$.

\section{Tiroides y receptores}

La detección temprana y masiva del hipotiroidismo congénito mediante la TSH neonatal ha favorecido las pesquisas en el campo de su etiología, permitiendo a los científicos estudiar su origen. Así se han visto casos de resistencia a la TRH en los receptores de TSH, u otros de relativa mayor frecuencia debidos a mutaciones en el gen del receptor $\beta$ de la hormona tiroidea. El receptor mutado, mediante un mecanismo de inhibición dominante, impide la unión de la triyodotironina a su receptor y da lugar a una menor respuesta tisular a la acción de las hormonas tiroideas. Recientemente se publicó un estudio de casos pertenecientes a dos familias colombianas, los primeros en ser informados en nuestro país. Los investigadores de la Universidad de los Andes nos muestran cómo la patología que afectaba a estos compatriotas recién nacidos fue encontrada gracias a los programas existentes sobre detección neonatal de enfermedades metabólicas congénitas como el hipotiroidismo neonatal $^{(24)}$.

\section{Enfermedad tiroidea autoinmune}

El desarrollo de la inmunogenética ha permitido conocer también los mecanismos fisiopatológicos de una patología común, la enfermedad de Graves-Basedow ${ }^{(25)}$. Esta condición es diagnosticada y tratada frecuentemente por los endocrinólogos de adultos, ya que, aunque se presenta en edades tempranas, su incidencia es baja en este grupo etario. El bocio difuso tóxico es una interesante enfermedad psicosomática, donde el estrés juega un papel desencadenante en la activación de un trastorno genéticamente determinado. Es mucho más común en mujeres que en hombres, y más frecuente en regiones con alto consumo de yodo. Como parte de las tiroidopatías autoinmunes (como la tiroiditis de Hashimoto), el bocio difuso tóxico es causado por estimulación del receptor de TSH localizado en la glándula tiroides, realizado por un anticuerpo contra ese receptor de TSH, lo que lleva a hiperplasia glandular y a hiperfunción ${ }^{(25)}$.
Por el contrario, la tiroiditis crónica autoinmune es causada por un anticuerpo que bloquea la estimulación por TSH y produce infiltración linfocitaria, bocio, bloqueos de ciertos pasos de la tiroxinogénesis (como la organificación del yodo) y finalmente hipotiroidismo. Cerca de una quinta parte de los pacientes con Graves-Basedow puede terminar espontáneamente con hipotiroidismo por una tiroiditis crónica subsiguiente, particularmente en pacientes que han logrado el eutiroidismo con tratamientos largos de propiltiouracilo, pero es casi la norma después del tratamiento con yodo radioactivo $^{(26,27)}$. La oftalmopatía está relacionada con una mediación inmunológica activada de los fibroblastos en músculos extraoculares, donde también hay receptores de TSH, y donde actúan los anticuerpos correspondientes ${ }^{(25)}$.

La autoinmunidad tiroidea la investigan los reumatólogos/ inmunólogos, como parte de las alteraciones en el sistema inmune que afectan el colágeno y los tejidos glandulares. Desde los primeros estudios de autoinmunidad hace más de medio siglo, se ha encontrado que una patología frecuente como la tiroiditis crónica de Hashimoto se asocia a otras enfermedades autoinmunes endocrinas menos frecuentes, y a diversas enfermedades del colágeno ${ }^{(28-33)}$. La determinación de anticuerpos contra las peroxidasas tiroideas se ha vuelto parte rutinaria del diagnóstico del hipotiroidismo subclínico ${ }^{(34,35)}$.

Hace 50 años, la tiroiditis de Hashimoto era considerada poco relevante en nuestro medio ${ }^{(36)}$. Sin embargo, en 1991 fue publicado un trabajo sobre cien casos de tiroiditis de Hashimoto $^{(37)}$, que despertó la conciencia entre los médicos de que en realidad esta era una patología frecuente en el país, que estaba presente en las dos terceras partes de los pacientes con bocio e hipotiroidismo, y que hizo necesaria la inclusión de la enfermedad en el diagnóstico diferencial de bocio hipercaptante en la gamagrafía realizada con m99Tc. El trabajo mereció el premio "Amigos de la Medicina". Al finalizar el siglo XX, la Asociación Colombiana de Endocrinología produjo un Consenso Colombiano para el Diagnóstico y Manejo de las Enfermedades Tiroideas, que tuvo mucha aceptación entre las especialidades relacionadas, facultades de medicina, centros asistenciales, profesores y estudiantes de pre y posgrado ${ }^{(38)}$.

Estos conceptos nos muestran cómo se diferencia claramente la endocrinología clínica de la molecular. Los clínicos podemos participar con casos, con estudios epidemiológicos, o con un buen conocimiento de la literatura, pero son otros los investigadores que van desarrollando el increíble conocimiento sobre las enfermedades hormonales, en particular las tiroideas.

\section{Cáncer de tiroides}

Actualmente, los investigadores colombianos se concentran en temas como cáncer de tiroides, epidemiología, cirugía y patología ${ }^{(39-50)}$. El cáncer de tiroides en Colombia tiene una 
incidencia calculada para mujeres en 2008 de 7,9 por 100.000 y para hombres fue de 0,8 por $100.000^{(39)}$. Entre 2003 y 2007 se presentaron 1.145 casos nuevos en el Instituto Nacional de Cancerología, que fueron clasificados por su tipo histopatológico en relación con la frecuencia, en: papilar (88\%), folicular (3\%), medular (3\%), de células de Hürtle (1\%), anaplásico (1\%) y otros, o no clasificados (4\%). Se calcula que anualmente aparecen 1.600 casos nuevos en todo el país. Nuevas técnicas quirúrgicas para cáncer se han diseñado en la Universidad de la Sabana, en Chía, Cundinamarca, donde también se han realizado metaanálisis sobre el tema ${ }^{(42-46)}$. De este grupo, un reciente estudio de costo-beneficio favorece la realización de tiroidectomías sobre hemitiroidectomías en neoplasias foliculares, excepto en casos de alto riesgo de hipoparatirodismo residual o de lesión del nervio recurrente laríngeo ${ }^{(49)}$. Esto está además en concordancia con la tendencia observada en las últimas décadas de hacer tiroidectomías oncológicas (o citorredución quirúrgica agresiva) en casos de carcinoma papilar, porque, a pesar de su buen pronóstico, se trata de cánceres de origen multicéntrico, con un número no despreciable de recidivas en casos de hemitiroidectomía, y en una cuarta parte de los casos, con variantes histológicas agresivas. La experiencia del grupo multidisciplinario de la Fundación Cardioinfantil de Bogotá, con cerca de medio millar de casos, justificó estas tiroidectomías oncológicas (que incluían en muchos casos vaciamientos centrales y laterales), por la presencia de los factores antes mencionados y en un número de casos, la falta de un adecuado seguimiento de los pacientes ${ }^{(50)}$.

\section{Estudios en grupos especiales}

Se han realizado estudios tiroideos en diversos grupos especiales. Por ejemplo, se ha confirmado la mayor incidencia de hipotiroidismo subclínico en pacientes con VIH/SIDA, aunque al parecer no hay una relación inmunológica ${ }^{(51)}$. Buscando valores autóctonos (verdaderos valores de referencia locales), se determinaron los valores de los marcadores tiroideos T3, T4 y TSH en comunidades indígenas y negras de Colombia. Se evaluaron 180 muestras de plasma, correspondientes a cinco grupos indígenas, tres grupos negros y un grupo control de mestizos de Santafé de Bogotá, utilizando las técnicas de inmunoensayo por polarización de la fluorescencia para T4 y de inmunoensayo en micropartículas enzimáticas para $\mathrm{T} 3 \mathrm{Y} \mathrm{TSH}^{(52)}$. En un estudio hecho en Bogotá sobre 447 mujeres posmenopáusicas se encontró una prevalencia de TSH elevada en $26.6 \%{ }^{(53)}$. Un estudio similar se hizo en población general ${ }^{(54)}$.

Investigadores de la Universidad de la Sabana están valorando los diferentes ejes hipotálamo-hipófisis-órgano periférico, y se ha investigado el efecto de la hormona tiroidea en el eje correspondiente a las suprarrenales ${ }^{(55,56)}$ y la relación de las diferentes hormonas en el estrés. Los pacientes hipertiroideos mostraron hiperactividad de ese eje, con origen central y respuestas de cortisol reducidas, que revirtieron con el trata- miento con carbimazol, mostrando que las hormonas tiroideas juegan un papel modulador en el eje hipotálamo-hipófisis-suprarrenales.

En las enfermedades psiquiátricas se han observado alteraciones endocrinas de los ejes hipotálamo-hipofisiarios, como el de las suprarrenales y el tiroideo ${ }^{(57,58)}$. También se está estudiando el papel que juega el hipotiroidismo subclínico en las depresiones mayores, y en la práctica no es infrecuente que se utilice la suplencia tiroidea para amplificar la respuesta de los antidepresivos.

El síndrome del enfermo eutiroideo ha sido motivo de dos publicaciones. Sin embargo, teniendo en cuenta la complejidad de la situación que genera demasiadas variables y la falta de estudios comparativos (tratados versus no tratados) entre otras falencias, las conclusiones sobre la bondad del tratamiento de suplencia generan más preguntas que respuestas ${ }^{(59)}$.

La administración de suplementos tiroides en niños con talla baja — rutinaria en la segunda mitad del siglo XX - ha caído en desuso, excepto en los casos con hipotiroidismo comprobado $^{(60)}$

El efecto de las hormonas tiroideas sobre el corazón ha sido ocasionalmente estudiado(61). También se continúan realizando las mediciones periódicas de la disponibilidad de sal yodada en diferentes regiones del país ${ }^{(62)}$. Un grupo multidisciplinario de especialistas estudió una serie de casos con encefalitis autoinmune de Hashimoto ${ }^{(63)}$. Jubiz, en Cali, demostró el efecto benéfico de la coadministración de vitamina $\mathrm{C}$ con levotiroxina en pacientes hipotiroideos con gastritis asociada y cambios en el pH gástrico, casos en los cuales el control del hipotiroidismo puede ser difícil(64).

También se han venido informando casos clínicos de rara ocurrencia que dan luz sobre ciertos mecanismos fisiopatológicos, acompañados de revisiones del tema ${ }^{(65-71)}$. Físicos, bioquímicos y patólogos también intervienen en los aportes colombianos $^{(72-74)}$. Es de anotar que el papel de los endocrinólogos clínicos en la investigación de las tiroidopatías se ha venido diluyendo - como ocurre también en otras especialidadesen cuanto a que ahora son personas con doctorados, expertos en biología molecular, las que realizan investigaciones hormonales. Nuevas sub-especialidades endocrinas que se dedican a grupos y patologías especiales (pediatras, ginecólogos, oncólogos, cirujanos, genetistas y especialistas en medicina nuclear) se han unido a la clásica endocrinología clínica como rama de la medicina interna. A partir de la primera Sociedad (Asociación) Colombiana de Endocrinología, se han generado otras sociedades científicas afines que desempeñan un papel importante en la educación médica, en la investigación y en la coordinación de los diferentes grupos afiliados a las universidades.

\section{Conclusiones}

La producción científica en el tema de la tiroides en Colombia es baja, no sólo al compararla con países desarrollados (Es- 
tados Unidos, 2.675, Países de Europa 4.980), sino con otros países de América Latina como Brasil (1.265), México (409), Argentina (448) y Chile (222). Sin embargo, Colombia tiene más publicaciones indexadas en PubMed que Venezuela, los demás países andinos, los dos países pequeños del Cono Sur y los de Centro América, Cuba, Puerto Rico y del Caribe. Hay que anotar que los endocrinólogos hemos sido desplazados en parte como autores de trabajos investigativos, y en su lugar aparecen cirujanos oncólogos, reumatólogos inmunólogos, salubristas, genetistas, patólogos y biólogos moleculares. La participación del endocrino (pediatra, internista o ginecólogo) se continuará presentando como parte de grupos multidisciplinarios de investigación.

Es necesario fomentar la investigación, lograr una mejor interacción entre organismos públicos y privados, mayor cooperación académica, esto con el fin de disminuir las brechas existentes, incrementar la publicación científica y aplicar los conocimientos generados en el propio país para mejorar las acciones epidemiológicas en los diferentes aspectos de la enfermedad tiroidea.

\section{Referencias}

1. Boussingault JB. Viajes científicos a los Andes ecuatoriales o colección de memorias sobre física, química e historia natural de la Nueva Granada, Ecuador y Venezuela. Traducción de J. Acosta. París, Lasserre, 1849. Citado por Rueda-Williamson R., Pardo-Téllez F. en La Prevención del bocio endémico en Colombia. Bol Ofic Sanit Panam 1966; Dic., 495-503.

2. Sociedad Colombiana de Endocrinología. La Tiroidología en Colombia. (A. Jácome, Editor). Ediciones Avanzada, Bogotá.1978.131 páginas.

3. Zúñiga S, Sanabria A. Prophylactic central neck dissection in stage N0 papillary thyroid carcinoma. Arch Otolaryngol Head Neck Surg. 2009;135 (11):1087-91.

4. Ucrós A, Hernández E, Acosta S. Historia de la Endocrinología en Colombia. 1999.

5. Jácome A. Historia de las Hormonas. Academia Nacional de Medicina, 2008.

6. Callejas L, Cortázar-García J, Gómez Afanador J, Gutiérrez A, Mendoza Hoyos H, Mendoza C, Reyes-Leal B., Sánchez Medina M, Tobar L, Ucrós-Cuéllar A, Arteaga M. Contribución al estudio de las endemias colombianas. Análisis de posibles factores etiológicos de cretinismo endémico. Premio de Ciencias 1959, Fundación Alejandro Ángel Escobar.

7. Gaitán-Marulanda E. Distribución, naturaleza y fuentes de origen de los agentes bociogénicos en el occidente colombiano. Premio de Ciencias 1976, Fundación Ángel Escobar.

8. Gaitán E, Cooksey RC, Matthews D, Presson R. In vitro mea- surement of antithyroid compounds and environmental goitrogens. J Clin Endocrinol Metab. 1983; 56(4):767-73.

9. Gait-an E, Cooksey RC, Legan J, Cruse JM, Lindsay RH, Hill J. Antithyroid and goitrogenic effects of coal-water extracts from iodine-sufficient goiter areas. Thyroid. 1993 spring; 3(1):49-53.

10. Vargas Uricoechea $\mathrm{H}$, Sierra- Torres $\mathrm{CH}$, Holguín-Betancourt CM, Cristancho-Torres L. Trastornos asociados a la deficiencia de yodo. Vigilancia permanente es deficitaria en zonas vulnerables. Medicina (Bogotá). 2012; 34(2): 119-145.

11. Otero Ruiz E. La medicina nuclear en Colombia, temprana historia y reminiscencia personales. 2002.

12. Patiño JF. Revisión histórica sobre el bocio en Suramérica y la Nueva Granada. Medicina (Bogotá).2001; 23(56):135150.

13. Cortázar J, Ahumada JJ, Otero E. Yodo radioactivo en fisiología y patología. Rev Soc Colomb Endocrinol 1996; 4:9-54.

14. Wahner HW, Gaitán JE, Escallón H. El tratamiento del hipertiroidismo en bocio nodular y Enfermedad de Graves con yodo radioactivo. Rev Soc Colomb Endocrinol. 1968; 6: 25-30.

15. Gaitán E, Cooksey RC, Meydrech EF, Legan J, Gaitan GS, Astudillo J, Guzman R, Guzman N, Medina P. Thyroid function in neonates from goitrous and nongoitrous iodine-sufficient areas. J Clin Endocrinol Metab. 1989; 69(2):359-63.

16. Ministerio de la Protección Social. Resolución número 00412 de 2000. Diario Oficial. 2000; 43956:1-216.

17. Carrillo, J.C. Detección de Hipotiroidismo Congénito en Colombia. Acta pediátrica colombiana. 1986. 4(1):31-37.

18. Castro Rojas, D. L. (2010). Evaluación del neurodesarrollo de pacientes con diagnóstico de hipotiroidismo congénito entre 0 a 15 años, encontrados en programas de tamización neonatal en Bogotá. Iatreia, 2010; 23(4-S),S-39.

19. Bernal J, Tamayo ML. La importancia del tamizaje neonatal. Pediatría. 1997; 32(2):137-139.

20. De Bernal MM, Bonilla RD, Caldas M, Chamorro GA, Matallana A. Tamización para hipotiroidismo congénito en Cali y constitución de un centro piloto de referencia para la identificación temprana de la enfermedad. Colomb Med 2003; 34:40-46.

21. Borrajo GJ. Newborn screening in Latin America at the beginning of the 21st century. J Inherit Metab Dis. 2007; 30(4):466-81

22. Hernández de Calderón LS, Bermúdez A. Tamizaje neonatal de hipotiroidismo congénito en Colombia: Estudio para establecer el punto de corte para el valor de TSH. Grupo de Genética Salud Pública, Laboratorio Nacional de Referencia. Epidemiología INS. IQEN. 2001; 6(15)229-230.

23. Salinas S, Ching B, Bermúdez A. Aplicación de Tamizaje para hipotiroidismo congénito en los recién nacidos de las IPS del Distrito de Bogotá entre Enero y Diciembre de 2001. Biomédica. 2004; 22(supl 1):81. 
24. Durán P, Mejía L, Lozano MC, Salguero F, Laverde G, Lattig MC. Resistencia a la hormona tiroidea en dos familias colombianas. Identificación y caracterización de mutaciones en el receptor beta de la hormona tiroidea. Medicina (Bogotá). 2013; 35(1):7-16.

25. Vargas-Uricoechea H, Sierra-Torres CH, Meza-Cabrera IA. Enfermedad de Graves-Basedow. Fisiopatología y Diagnóstico. Medicina (Bogotá). 2013;35(1):41-66.

26. Cadavid L, Vivas J, Medina R. Conversión a hipotiroidismo en tratamiento con I-131 por hipertiroidismo secundario a enfermedad de Graves, Hospital de San José, enero 2005 diciembre 2008. Repert med cir 2009; 18(4):231-236.

27. Builes CA. Enfoque del paciente con enfermedad de graves revisión. Rev Med 2005; 13(1):93-98.

28. Anaya JM, Castiblanco J, Rojas-Villarraga A, Pineda-Tamayo $\mathrm{R}$, Levy RA et al. The multiple autoimmune syndromes. A clue for the autoimmune tautology. Clin Rev Allergy Immunol. 2012; 43(3):256-264.

29. Anaya JM, Tobon GJ, Vega P, Castiblanco J. Autoimmune disease aggregation in families with primary Sjögren's syndrome. J Rheumatol. 2006; 33(11):2227-2234.

30. Anaya JM, Castiblanco J, Tobón GJ, García J, Abad V et al. Familial clustering of autoimmune diseases in patients with type 1 diabetes mellitus. J Autoimmun. 2006; 26(3):208214.

31. Hudson M, Rojas A, Coral P, López S, Mantilla RD, Chalem P, Canadian Scleroderma Research Group, Colombian Scleroderma Research Group, Baron M, Anaya JM. Polyautoimmunity and familial autoimmunity in systemic sclerosis. J Autoimmun. 2008; 31(2):156-159.

32. Roman-Gonzalez A, Moreno ME, Alfaro JM, Uribe F, LatorreSierra G, Rugeles MT, Montoya CJ. Frequency and function of circulating invariant NKT cells in autoimmune diabetes mellitus and thyroid diseases in Colombian patients. Hum Immunol. 2009; 70(4):262-8

33. Cárdenas Roldán $\mathrm{J}^{1}$, Amaya-Amaya J, Castellanos-de la Hoz J, Giraldo-Villamil J, Montoya-Ortiz G, Cruz-Tapias P, RojasVillarraga A, Mantilla RD, Anaya JM. Autoimmune thyroid disease in rheumatoid arthritis: a global perspective. Arthritis. 2012 (2012), Article ID 864907. http://dx.doi. org/10.1155/2012/864907.

34. Londoño AL, Gallego ML, Bayona A, Landázuri P. Hypothyroidism prevalence and its relationship to high levels of thyroid peroxidase antibodies and urinary iodine in a population aged 35 and over from Armenia, 2009-2010. Rev Salud Publ (Bogota). 2011; 13(6):998-1009.

35. Escobar M, Villamil M, Ruiz O. Prevalencia de anticuerpos antiperoxidasa y antitiroglobulina en jóvenes con hipotiroidismo subclínico y clínico. Medicina \& Laboratorio, 2011; 17, 351-57.

36. Jácome-Roca A, Mesa Arévalo A. Autoanticuerpos a la tiroglobulina en tiroidopatías. Univ Med 1964; 6:43-50.
37. Escobar I, Kattah W, Niño A, Acosta E, Saavedra C, Ucrós A. Tiroiditis de Hashimoto, estudio de 100 casos. Acta Med Colomb 1991; 16:18-29.

38. Asociación Colombiana de Endocrinología. Consenso Colombiano para el Diagnóstico y Manejo de las Enfermedades Tiroideas. Acta Med Colomb 1999; 24:159-174.

39. Colombia. Instituto Nacional de Cancerología, Anuario Estadístico, Bogotá, Noviembre de 2009.

40. Chala AI, Franco HI, Aguilar CD, Cardona JP. Estudio descriptivo de doce años de cáncer de tiroides, Manizales, Colombia. Rev Colomb Cir. 2010; 25:276-89.

41. Bravo LE, Collazos T, Collazos P, García LS, Correa P. Trends of cancer incidence and mortality in Cali, Colombia. 50 years experience. Colomb Med 2012; 43(4):246-255.

42. Sanabria A, Zúñiga S. Carcinoma papilar de tiroides en niños y adolescentes: relación de las características patológicas con la recurrencia. Rev Colomb Cir 2007; 22(4):202208.

43. Alfonso E, Sanabria A, Castillo M. Surgeons overestimate the risk of malignancy in thyroid nodules, evaluation of subjective estimates using a bayesian analysis. Biomedica. 2011; 31(4):590-598.

44. Sanabria A, Domínguez LC, Vega V, Osorio C. Prognosis of patients with thyroid cancer who do not undergo surgical treatment: a SEER database analysis. Clin Transl Oncol. 2011; 13(9):692-696.

45. Sanabria A, Carvalho AL, Piana de Andrade V, Pablo Rodrigo J, Vartanian JG, et al. Is galectin-3 a good method for the detection of malignancy in patients with thyroid nodules and a cytologic diagnosis of "follicular neoplasm"? A critical appraisal of the evidence. Head Neck. 2007; 29(11):1046-54.

46. Sanabria A, Carvalho AL, Silver CE, Rinaldo A, Shaha AR et al. Routine drainage after thyroid surgery--a meta-analysis. J Surg Oncol. 2007; 96(3):273-80.

47. Chala AI, Pava R, Franco HI, Alvarez A, Franco A. Criterios ecográficos diagnósticos de neoplasia maligna en el nódulo tiroideo: Correlación con la punción por aspiración con aguja fina y la anatomía patológica. Rev Colomb Cir 2013; 28(1):15-23.

48. Cadena E, Bastidas F, Angarita E, Garzón JG. Resección de recaídas de cáncer diferenciado de tiroides mediante cirugía radioguiada. Rev Col Cancerol 2012; 16(2):130-134.

49. Corso C, Gomez X, Sanabria A, Vega V, Dominguez LC, Osorio C. Total thyroidectomy versus hemithyroidectomy for patients with follicular neoplasm. A cost-utility analysis. Int J Surg. 2014; 12(8):837-42. doi: 10.1016/j. ijsu.2014.07.005. Epub 2014 Jul 11.

50. Sánchez G, Gutiérrez C, Valenzuela A, Tovar JR. Carcinoma diferenciado de la glándula tiroidea: hallazgos en 16 años de manejo multidisciplinario. Rev Colomb Cir. 2014; 29:102-109

51. Gómez CH, Vesga JF, Lowenstein E, Suárez JO, Gil FA et al. 
Detección de hipotiroidismo en un programa de atención de VIH/SIDA en un hospital de Bogotá, Colombia. Rev Chil Infectol. 2011; 28(1):59-63.

52. Bernal-Villegas JE, Martínez JC, Gómez-Gutiérrez A, Pineda $\mathrm{S}$, Vargas. Marcadores Tiroideos en comunidades indígenas y negras de Colombia. Univ Med 1995; (4):124-129.

53. Barón Castañeda G. Prevalencia de hipotiroidismo subclínico en la población post-menopáusica. Rev Col Menopaus 2001;7(2).

54. Builes CA, Rosero 0, García J. Evaluación de disfunción tiroidea según TSH en una población de Bogotá. Acta Med Colomb 2006; 31: 66-70.

55. Lizcano F, Rodríguez JS. Thyroid hormone therapy modulates hypothalamic-pituitary-adrenal axis. Endocr J. 2011; 58(2):137-42.

56. Lizcano F, Salvador J. Effects of different treatments for hyperthyroidism on the hypothalamic-pituitary-adrenal axis. Clin Exp Pharmacol Physiol. 2008 ;35(9):1085-90

57. Castilla-Puentes R, Secin R, Grau A, Galeno R, De Mello MF, Castilla-Puentes S, Castilla-Puentes W, Sanchez-Russi CA. A multicenter study of bipolar disorder among emergency department patients in Latin-American countries. Int J Psychiatry Med. 2011; 42(1):49-67.

58. Machado-Alba JE, Plaza CD, Solarte Gómez MJ. [Antidepressant prescription patterns in patients affiliated with the General Social Security Health System of Colombia]. Rev Panam Salud Pública. 2011 Nov; 30(5):461-8.

59. Trigos-Pallares PL. Suplementación de hormona tiroidea en pacientes pediátricos críticos con síndrome eutiroideo enfermo. 2010. http://repository.urosario.edu.co/bitstream/handle/10336/1657/88282214.pdf?sequence=1

60. Hernández-Cassis C, Cure-Cure C, López-Jaramillo P. Effect of thyroid replacement therapy on the stature of Colombian children with minimal thyroid dysfunction. Eur J Clin Invest. 1995; 25(6):454-6.

61. Vargas EA, González JD, Pérez MT, Granados CE, Gómez EA. Comportamiento de la TSH y la T4 en una cohorte de pacientes con arritmia cardiaca tratados con amiodarona $\mathrm{u}$ otros antiarrítmicos. Rev Colomb Cardiol. 2008; 15(4) 161-164.

62. Ruiz, Hugo; Jiménez, Guillermo. Prevalencia de los desórdenes por deficiencia de yodo e ingestión promedio de sal Colombia, 1994-1998 / Bogotá; INS; nov. 2001. 117 p.
63. Sánchez Contreras A, Rojas SA, Manosalva A, Méndez Patarroyo PA, Lorenzana P, Restrepo JF, Iglesias-Gamarra A, Rondon F. Hashimoto encephalopathy (autoimmune encephalitis). J Clin Rheumatol. 2004; 10(6):339-43.

64. Jubiz W, Ramirez M. Effect of vitamin C on the absorption of levothyroxine in patients with hypothyroidism and gastritis. J Clin Endocrinol Metab. 2014 Jun; 99(6):E1031-4. doi: 10.1210/jc.2013-4360. Epub 2014 Mar 6.

65. Romero-Rojas A, Bella-Cueto MR, Meza-Cabrera IA, Cabezuelo-Hernández A, García-Rojo D, Vargas-Uricoechea H, Cameselle-Teijeiro J. Ectopic Thyroid Tissue in the Adrenal Gland: Report of 2 cases with pathogenetic implications. Thyroid. 2013 Mar 19. [Epub ahead of print] Clin Exp Pharmacol Physiol. 2008 Sep; 35(9):1085-90.

66. Céspedes C, Duran P, Uribe C, Chahín S, Lema A, Coll M. Thyroid abscess. A case series and literature review. Endocrinol Nutr. 2013; 60(4):190-196.

67. Aschner P, Jácome A. Tiroides ectópico: informe de cuatro casos. Acta Med Colomb. 1984; 9(6):310-155.

68. Rozo DF, Yurgaky J, Polanía D y cols. Cáncer de tiroides en nódulo hipercaptante por gammagrafía, serie de casos. Rev Fac Med U Nal, 2010; 18(2).

69. Baena J, Gutiérrez J, Redondo K, Redondo C. Estrumosis peritoneal: reporte de caso y revisión de la literatura. Rev Colomb Obstet Ginecol, 2011 Dic; 62(4).

70. De la Calle, Y. (1997). Tumores de células de Hürthle de la glándula tiroides: 28 casos. Hospital Universitario del Valle, Cali. Colombia Médica, 28(1), 16-21.

71. Bonnet Palencia, I. I., Benedetti Padrón, I., \& Sáenz Pupo, J. C. (2010). Gammagrafía con receptores de somatostatina en un caso de carcinoma medular de tiroides. AJ48-6. Alasbimn Journal, 12, 48

72. Puerta A, Morales J, Díaz G. Simulador de tiroides de adulto. Rev Soc Col Física. 2006; 38(2):938-941.

73. Echeverri NP, Ortiz BL, Caminos JE. (2010). Proteomic analysis of primary cultures from thyroid. Rev Col Quim; 2010; 39(3):343-358.

74. Romero-Rojas AE, Diaz-Perez JA, Mastrodimos M, Chinchilla SI. Follicular thyroid carcinoma with signet ring cell morphology: fine-needle aspiration cytology, histopathology, and immunohistochemistry. Endocr Pathol. 2013; 24 (4): 239-45. doi: 10.1007/s12022-013-9271-x 